EUROPEAN ORGANIZATION FOR NUCLEAR RESEARCH

European Laboratory for Particle Physics

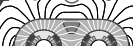

(c) (0)

Large Hadron Collider Project

LHC Project Report 146

\title{
Thermohydraulics of resistive transitions of the LHC prototype magnet string: Theoretical modeling and experimental results
}

\author{
M. Chorowski, B. Hilbert, L. Serio, R. van Weelderen
}

\begin{abstract}
In preparation for the Large Hadron Collider (LHC) project, a $40 \mathrm{~m}$-long prototype superconducting magnet string, representing a half-cell of the machine lattice, has been built and operated. The superconducting magnets which comprise this string normally operate in a pressurized static bath of superfluid helium at a pressure of 1 bar and at a temperature of 1.9 K. At $13.1 \mathrm{kA}$ they have about $15.3 \mathrm{MJ}$ of stored magnetic energy. A series of tests was performed to assess the thermohydraulics of resistive transitions (quenches) of the string of magnets. These measurements provide the necessary foundation for describing of the observed pressure rise as the combination of two processes, each acting on a different time scale. The measurements are presented and an explanatory model description of the events is given.
\end{abstract}

CERN - LHC-ACR Division

CEC-ICMC'97 - Portland - OR - USA

July 28th - August 1st, 1997

Administrative Secretariat

LHC Division

CERN

$\mathrm{CH}$ - 1211 Geneva 23

Switzerland

Geneva, 6 October 1997 


\title{
THERMOHYDRAULICS OF RESISTIVE TRANSITIONS \\ OF THE LHC PROTOTYPE MAGNET STRING: \\ THEORETICAL MODELING AND EXPERIMENTAL RESULTS
}

\author{
M. Chorowski, B. Hilbert, L. Serio and R. van Weelderen \\ LHC Division, CERN \\ CH-1211 Geneva 23, Switzerland
}

\begin{abstract}
In preparation for the Large Hadron Collider (LHC) project, a $40 \mathrm{~m}$-long prototype superconducting magnet string, representing a half-cell of the machine lattice, has been built and operated. The superconducting magnets which comprise this string normally operate in a pressurized static bath of superfluid helium at a pressure of 1 bar and at a temperature of 1.9 K. At $13.1 \mathrm{kA}$ they have about $15.3 \mathrm{MJ}$ of stored magnetic energy. A series of tests was performed to assess the thermohydraulics of resistive transitions (quenches) of the string of magnets. These measurements provide the necessary foundation for describing of the observed pressure rise as the combination of two processes, each acting on a different time scale. The measurements are presented and an explanatory model description of the events is given.
\end{abstract}

\section{INTRODUCTION}

With the magnet "cold mass" we designate the coils, the aluminum collars and iron yokes, the helium in which the whole is immersed, and the stainless-steel vessel around the assembly. When a magnet in the string quenches, the coils in the whole string are driven resistive by dedicated heaters and the current is ramped down with a decay constant of $250 \mathrm{~ms}$. Consequently its full stored magnetic energy is dissipated by ohmic heating in the coils. ${ }^{1}$ This heat will eventually end up in the cold mass, and unless the helium contained inside the cold mass is expelled, it will drive the pressure an order of magnitude beyond the $2 \mathrm{MPa}$ (20 bar) design pressure of the vessel. Therefore a quench relief valve (QRV) must be opened, to allow the helium to discharge and to limit the pressure rise to below the $2 \mathrm{MPa}$ limit.

A series of tests was performed on a $40 \mathrm{~m}$-long prototype superconducting magnet string, representing a half-cell of the LHC machine lattice. These tests were aimed particularly at obtaining an understanding of the quench thermohydraulics, in order to enable the 


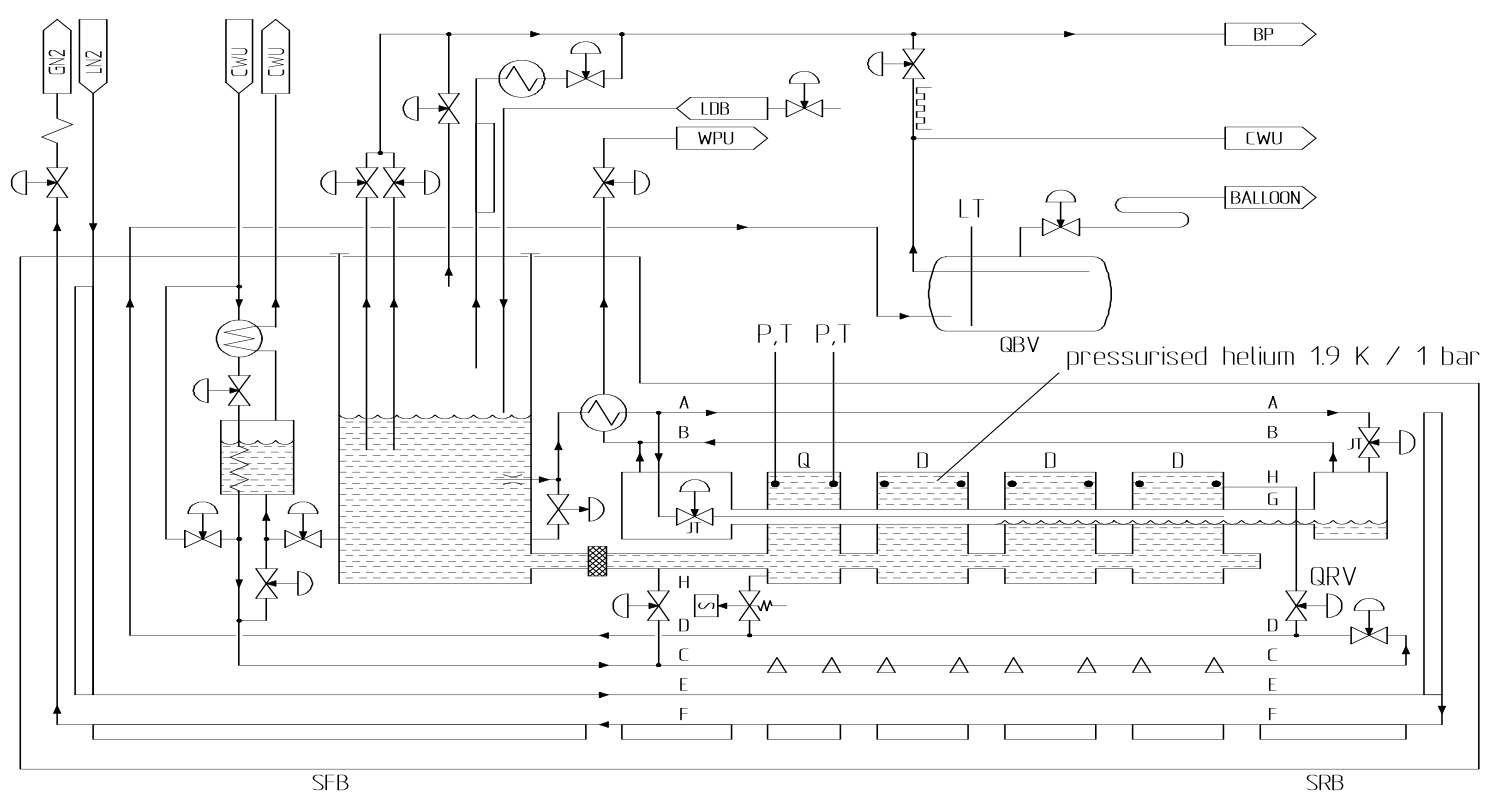

Figure 1. Schematic of the cryogenic configuration of the LHC prototype string.

numerical evaluation of possible other magnet string configurations, and to permit the sizing of crucial cryogenic safety equipment and helium recovery systems.

We will discuss the main measurements and deduce from them an explanatory model description of the observed pressure and temperature transients. ${ }^{2}$

\section{LHC TEST STRING CONFIGURATION}

\section{Installation Layout}

Figure 1 shows the schematic of the cryogenic configuration of the prototype string. It comprises one quadrupole magnet $(\mathrm{Q})$ and three dipole magnets (D), a cryogenic feed box (SFB) on the left end, and a return box (SRB) on the right end. The whole is mounted on a slope of $1.4 \%$, corresponding to the maximum slope that will be encountered in the LHC machine tunnel. The magnets normally operate in a pressurized static bath of superfluid helium at a pressure of 1 bar and at a temperature of $1.9 \mathrm{~K}$. The liquid helium content in the helium cold mass is about $20 \ell / \mathrm{m}$, which amounts to a total inventory of $850 \ell$. The four magnets of the string are hydraulically connected and this forms a single hydraulic unit. In case of overpressure the helium is discharged via a quench relief valve (QRV), mounted at the upper part of the rightmost dipole magnet. The helium then enters first the cold line D ( $20 \mathrm{~K}, 1$ bar), into an isolated recovery vessel and finally a warm storage balloon. The other circuits are for cooldown, $1.9 \mathrm{~K}$ temperature control, and cryostat heat intercepts. ${ }^{3}$

\section{Instrumentation}

The string is equipped with a full range of cryogenic instruments: temperature and pressure sensors, liquid level gauges and mass flowmeters. Of particular interest for quench analysis are the temperature sensors (Allen-Bradley $100 \mathrm{Ohm}$ carbon resistors) and piezoresistive cold pressure sensors (Siemens KPY16, Siemens KPY47), placed in the end-volumes of each of the magnet cold masses. 


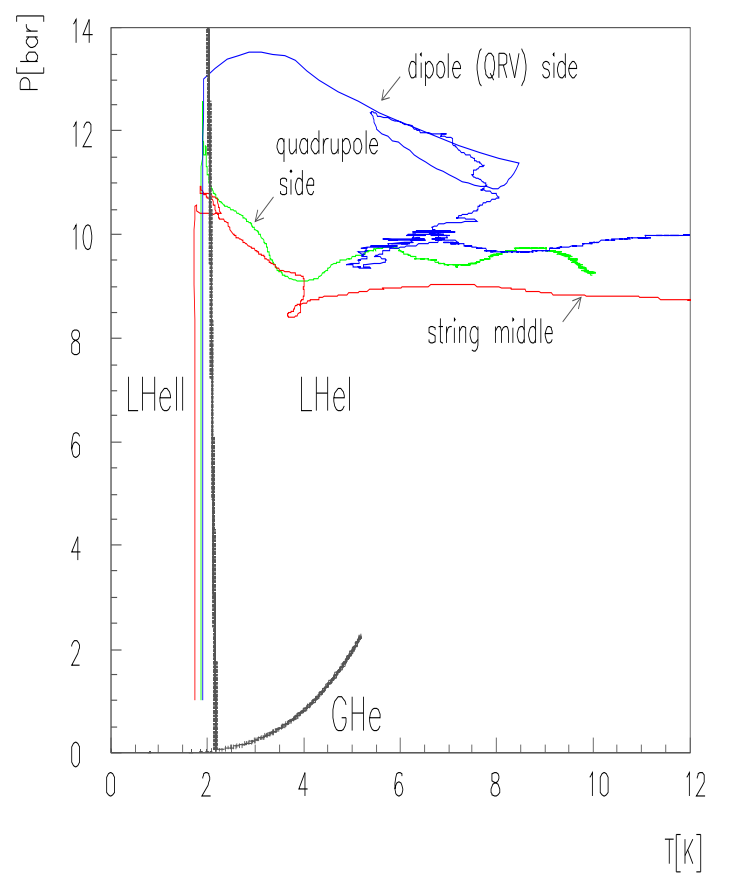

Figure 2. Phase diagram of the string cold mass helium after a quench.

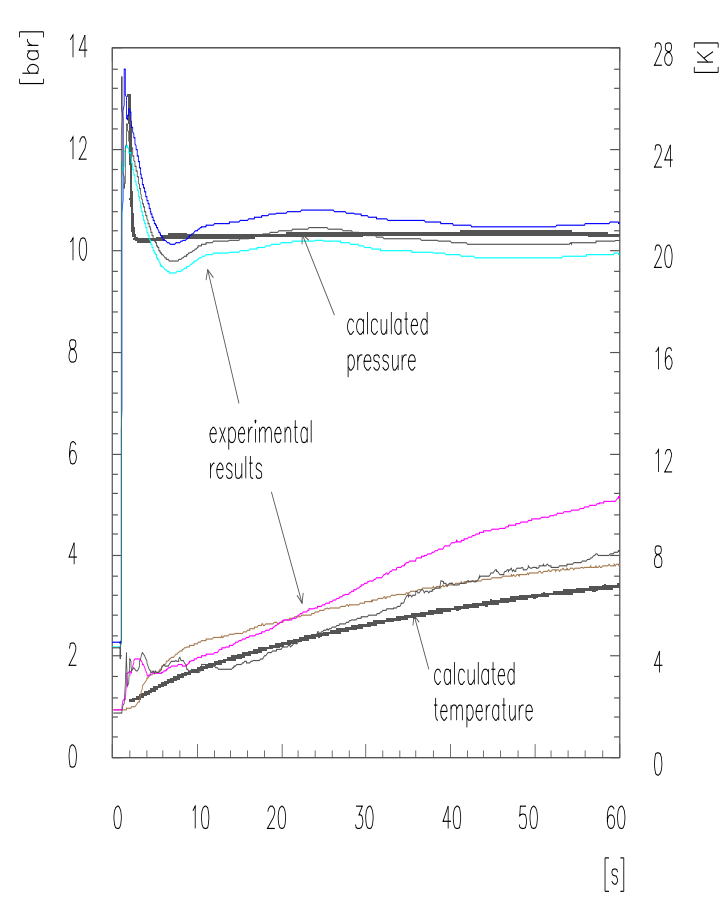

Figure 3. Measured development of the string cold mass helium after a $13.1 \mathrm{kA}$ quench, as well as the model simulation.

Data was recorded at a sampling rate of $1 \mathrm{~ms}$ with transient recorders using pre-trigger and post-trigger recording. ${ }^{4}$ The electrical quench detection signal was taken as trigger.

\section{Pressure Distribution after a Quench}

Prior to their mounting in the string each magnet has been quench tested seperately in a stand-alone configuration. ${ }^{5}$ Temperatures were measured in the end-volumes and midlength in the collar and yoke. Pressures were measured in the end-volumes, and at three locations along the length of the magnet in the collar noses. These measurements showed that the pressure development after a quench is homogeneous over the bulk of the magnet. In the string test only the pressure and temperature sensors in each of the end-volumes of each of the magnets are retained. Based on the previously demonstrated homogeneity of the pressures, they provide sufficient information to perform the analysis of the pressure rise processes and to establish the energy balance between magnets, helium inside and helium discharged from the cold masses.

\section{EVOLUTION OF THE HELIUM THERMODYNAMIC STATE AFTER A QUENCH}

\section{Pressure and Temperature}

Figures 2 and 3 show the development of the helium in the string cold mass after a quench. In the phase diagram of figure 2 the helium starts at the working point of $1.9 \mathrm{~K}$, 1 bar, it then moves steeply (within about $150 \mathrm{~ms}$ ) up in pressure up to about 14 bar while remaining at about the same temperature. The pressure drops then to about 10 bar and the temperature gradually increases. This last development where the helium evolves in the supercritical region is partly dictated by our choice of operating mode of the QRV. It serves as a pressure-regulating device, set at 10 bar.

In some experiments we have let the QRV function as a one-shot opening valve. In 


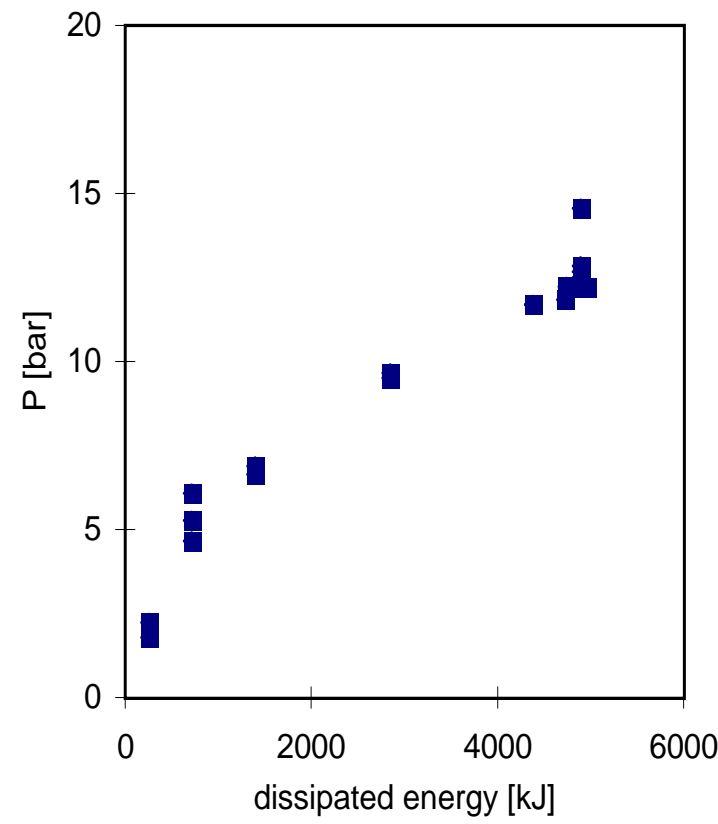

Figure 4. Pressure peak values from string measurements as function of dissipated energy in the coils.

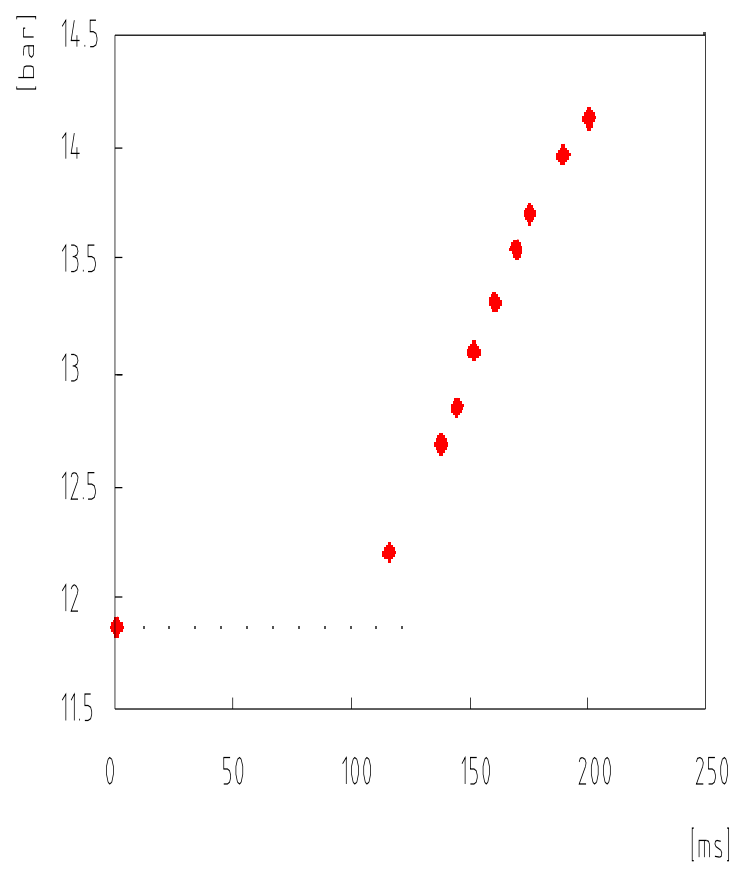

Figure 5. Pressure peak dependence on QRV opening time.

those cases, instead of evolving in the supercritical region, the working point moves to the saturation line. Two-phase flow might then limit the discharge capacity of QRV's by sonic constriction, complicates the analysis and makes any scaling of the results rather difficult. In addition, by remaining in the supercritical range, the overall heat exchange between the helium and the magnet structure is improved, since the uniformity of the supercritical phase allows the helium to make use of the maximum available area for heat exchange. More heat is then extracted from the cold mass, which shortens recooling times for the magnets. On these grounds we have therefore chosen, for further string tests as well as for the future LHC, to operate with the QRV's as pressure regulating devices only.

\section{Pressure Peak Dependence on Energy Dissipated in the Magnet Coils}

The height of the pressure peaks (fig. 3) which occur about $150 \mathrm{~ms}$ after the quench are related to the energy dissipated in the magnet coils (fig. 4). The same kind of relation, showing some slight differences between the different magnets, was observed when the magnets were tested in stand-alone configuration. ${ }^{5}$ In the string configuration, the heights of pressure peaks of neighbouring magnets tend to equalize. The extent to which magnets which are far apart influence each other, is determined by their separation in terms of sound propagation in helium.

\section{Pressure Peak Dependence on Valve Opening Time}

Figure 5 shows the height of the pressure peak as function of the QRV opening time. This time was varied from 120 to $220 \mathrm{~ms}$, with an additional measurement taken at $0 \mathrm{~ms}$ when we opened the QRV valve before triggering a quench. This clearly shows that the height of the pressure peak is inherent to the magnets and cannot be reduced to zero by any means of fast discharge. The influence of helium discharge is weak, and becomes appreciable only for opening delays greater then about $100 \mathrm{~ms}$. 


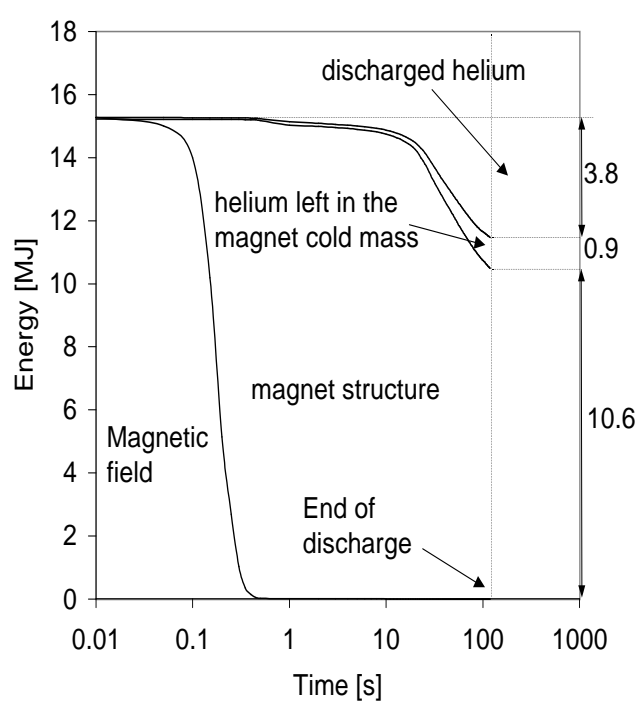

Figure 6. Time evolution of the distribution of energy over the magnetic field, magnet structure, cold mass helium, and discharged helium.

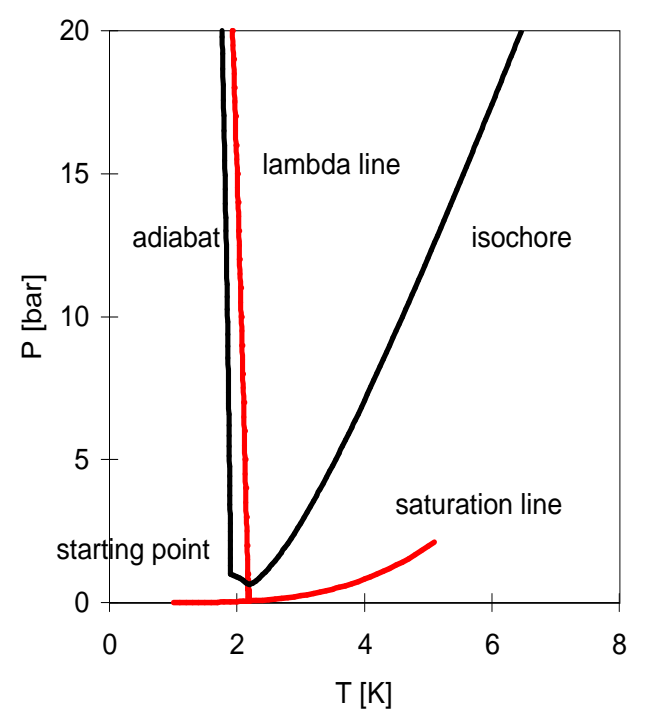

Figure 7. Ideal lines of evolution for isochoric heating, and adiabatic compression of helium.

\section{Energy Distribution}

The magnetic field energy is calculated from the measured current decay, common to all magnets, and the respective magnet inductances. The internal energy of the helium inside each of the cold masses is calculated from the temperatures and pressures measured in the end-volumes, whereby it is assumed that the helium thermodynamic state is homogeneous over half the cold masses adjacent to the measurement locations. The helium mass discharge and corresponding enthalpy flow is calculated from the QRV $K_{v}$ value, and the temperature and pressure measurement before and after the QRV. The energy needed to complete the sum of these energies up to the stored magnetic energy at the quench current level is attributed to the magnet structure.

Figure 6 shows the time evolution of the energy distribution. From the initial magnetic field energy at quench current, about $6 \%$ ends up in the helium in the cold mass, about $25 \%$ leaves the cryostat with the discharged helium, and the remainder ends up in the magnet structure.

The heating power of the helium inside the cold mass is calculated as the differential of its energy development minus the enthalphy outflow (fig. 9).

\section{Process Identification}

The foregoing observations have led us to identify the two main processes, each acting on a different time scale, which govern the helium thermohydraulics after a quench.

On a short time scale, up to roughly $150 \mathrm{~ms}$ after the quench, the bulk of the helium is adiabatically compressed. This follows directly from the measured evolution of the helium in the phase diagram as compared with ideal lines of evolution for isochoric heating, and adiabatic compression (fig. 7).

On a long time scale the bulk helium is heated at a relatively lower rate by the warm coils and the magnet structure, while simultaneously being allowed to discharge from the cold mass.

The existence of these two processes becomes clearly evident when in addition to the forementioned measurements, one observes the pressure evolution at much lower than usual 


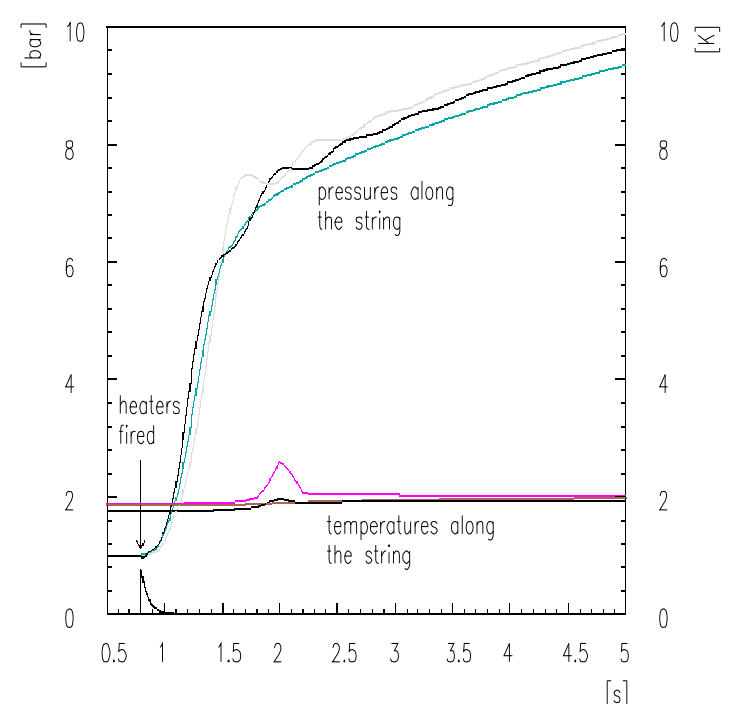

Figure 8. Development of the string cold mass helium after a low energy quench (at $5 \mathrm{kA})$.

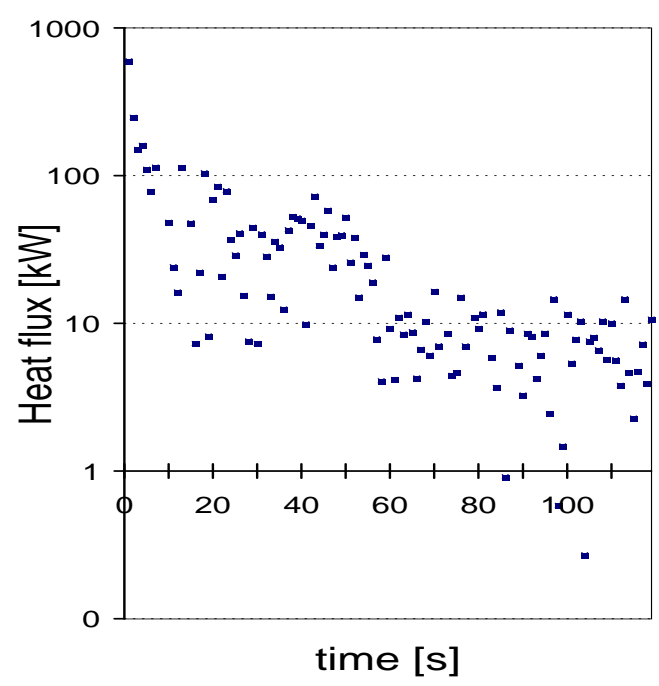

Figure 9. Power transfered from the magnet to the helium for a high energy quench (at $13.1 \mathrm{kA})$.

quench energies. The two distinct rates of pressure rise associated with respectively the short time scale (high rate) and long time scale (low rate), as well as the changeover between these two processes can easily be recognized in figure 8 .

\section{MODELS}

\section{Short Time Scale Model}

During about the first $150 \mathrm{~ms}$, the bulk of the helium $(\approx 200 \ell$ per magnet) undergoes an adiabatic compression. At this stage the heat originating from the dissipated magnetic energy is still concentrated in the magnet coils. The coils exchange heat efficiently only with the relatively small amount of helium with which they are in close thermal contact: roughly $2 \%$ of the total helium contents as estimated from micrograph photographs of assembled coil cross-sections. This helium would attain very high pressures if not given the possibility, via a sufficient radial venting connection to the bulk, to expand into the region occupied by the bulk helium. ${ }^{6}$ As a consequence the bulk helium gets adiabatically compressed. The, by now vapourized, helium surrounding the hot coils has become a limit to the heat transfer, which determines the end of the fast pressure rise.

One can thus distinguish: the (moderately) "confined" helium as being the fraction of helium in close thermal contact with the coils, the bulk helium, and the radial venting connection between the two. This connection must be sufficient, to prevent the development of high local pressures near the coils.

The adiabatic compression process is schematically depicted as a two-volume model ${ }^{7}$ in figure 10. The peak pressure is very sensitive to the final temperature of the expanding "confined" helium. For the prototype magnet string, the maximum coil temperature after a $13.1 \mathrm{kA}$ quench is about $140 \mathrm{~K}$. Simply assuming no temperature difference between the confined helium fraction, $2 \%$, and the coil would lead to peak pressure values of more than twice the measured values (fig. 11).

Assuming a 2\% confined helium fraction we find that the heat and corresponding power necessary to reproduce the observed pressure peaks are quite small, a few hundreds of kJ (1-2\% of the total magnetic energy) and about $1 \mathrm{MW}$, which is attained in our simulations 


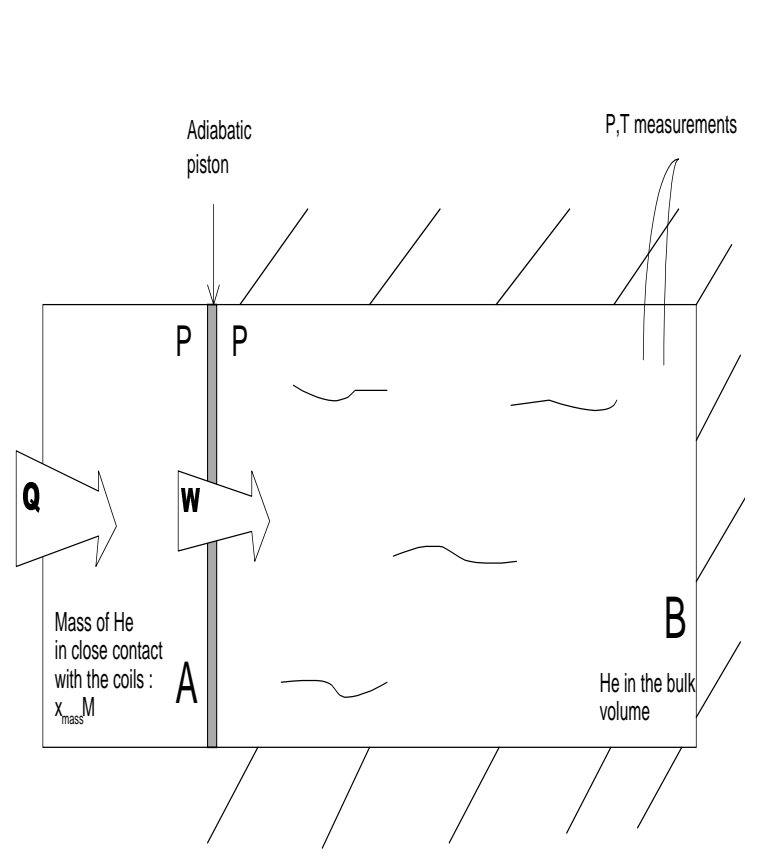

Figure 10. Two volume model of the adiabatic compression of the bulk helium (B) by the expanding confined helium (A).

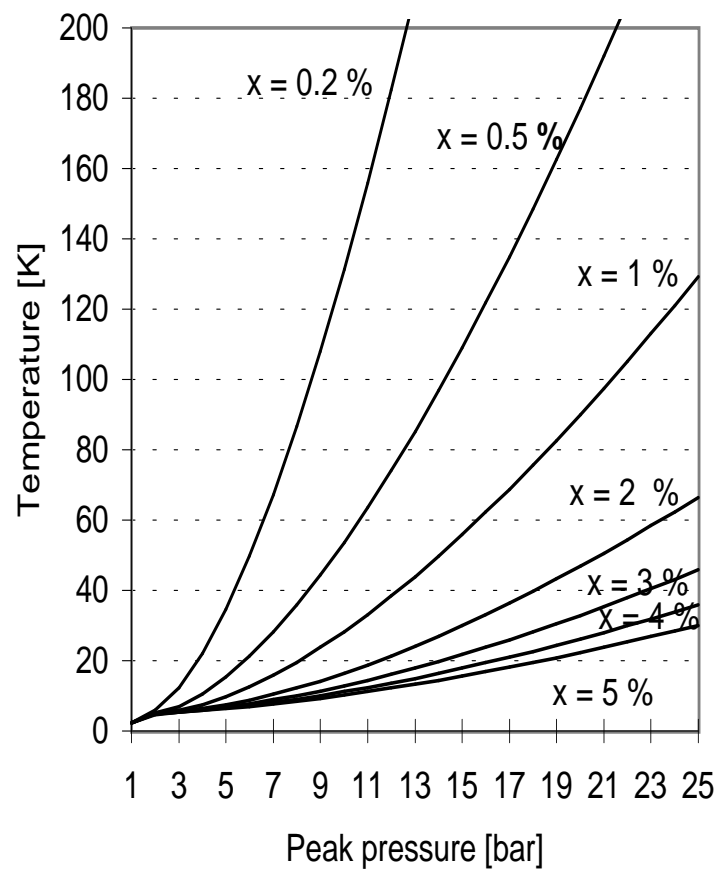

Figure 11. Calculated confined helium temperature necessary for compressing adiabatically the bulk helium to a given peak pressure; $x$-confined helium fraction.

with a heat exchange coefficient of the order of a few $\mathrm{W} / \mathrm{cm}^{2}$ and a temperature difference between the coils and the expanding helium of about $110 \mathrm{~K}$.

The height of the pressure peak depends on the transferred heat. This heat depends on the coil temperature, which in turn depends on the dissipation of magnetic energy. These links can explain the measured dissipated energy dependence of the pressure peak (fig. 4).

\section{Long Time Scale Model}

After the adiabatic compression has ended, the expanded helium and the compressed bulk helium mix, the pressure thereby drops from its peak value down to a few bar and the temperature increases by about $0.5 \mathrm{~K}$. At the same time heat in the coils is gradually being transferred by solid conduction to the collars and yoke. This way, the bulk helium gets exposed to an increasingly larger heat exchange area. At this stage we can consider the magnet structure as a heat source for the bulk helium. The helium warms up and pressurizes at a rate low enough such as to enable to keep its pressure below the cryostat design limits, by discharge through a QRV.

The heat and corresponding power necessary to reproduce the observed pressure and temperature development are quite small and can be easily accounted for by thermal convection.

\section{Model Comparison with Measured Data}

Based upon these two models, linked through a mixing phase, we are able to reproduce the measured data (fig. 3) using as input a curve fit of the measured heating power as shown in figure 9. 


\section{CONCLUSIONS}

The thermohydraulics of resistive transitions of the LHC prototype magnet string is governed by two main processes: initially by a fast adiabatic compression of the bulk of the helium by the rapidly expanding confined helium, and finally by a global convective heating of the bulk helium. The transition between these processes is formed by a mixing between the expanded helium and the compressed bulk helium.

This overall behaviour remains valid as long as the radial venting connection between the confined and bulk helium remains sufficient to allow the confined helium to expand into the region occupied by the bulk helium.

The initial pressure peaks due to the adiabatic compression of the bulk helium are inherent to the magnet design. According to the adiabatic compression model they will be es-

pecially sensitive to the amount of confined helium. These pressures are mediated by sound waves. Mass transport is thus not involved, which is why the initial peak pressures cannot be influenced below their inherent value by any means of fast discharge. The influence of helium discharge is weak, and becomes appreciable only for opening delays greater then about $100 \mathrm{~ms}$.

The measured quench pressure developments can be simulated with sufficient accuracy to justify extending the simulations to different string geometries. ${ }^{2}$

\section{REFERENCES}

1. F. Rodriguez-Mateos, G. Gerin and A. Marquis, Quench Protection Test Results and Comparative Simulations on the First 10 Metre Prototype Dipoles for the Large Hadron Collider, Fourteenth International Magnet Technology Conference (MT-14), Tampere, (1995).

2. B. Hilbert, Modeling and Experimental Analysis of the Thermohydraulics of Resistive Transitions on the LHC Prototype Magnet String, LHC Project Note 86 (1997).

3. A. Bézaguet, J. Casas Cubillos, B. Flemsäter, B. Gaillard-Grenadier, Th. Goiffon, H Guinaudeau, Ph. Lebrun, M. Marquet, A. Suraci, L. Tavian and R. van Weelderen, The Superfluid Helium Cryogenic System for the LHC Test String: Design, Construction and First Operation, Advances in Cryogenic Engineering Vol. 41 (1996), pp. 777-784.

4. D. Brahy, J. Casas Cubillos, M. Grippeling, D. Lavielle, G. Leo, L. Madaro, A. Rijllart, R. Saban, M. Skiadelli, The Control and Data Acquisition of the LHC Test String, International Conference on Accelerator and Large Experimental Physics Control Systems (ICALEPCS'95 Chicago, 1995).

5. G. Gerin, B. Vullierme and R. van Weelderen, Measurement of the Thermohydraulic Behaviour of LHC Dipole Prototypes after a Quench, Advances in Cryogenic Engineering Vol. 41 (1996), pp. 811-818.

6. Ph. Lebrun, Th. Wahlström, R. van Weelderen and L. Williams, Investigation of Quench Pressure Transients in the LHC Superconducting Magnets, Cryogenics 34 ICEC Supplement (1994), pp. 705-708. Genova, (1994).

7. Ph. Lebrun, Private Communication, (CERN). 